\title{
Modeling of non-Gaussian colored noise and application in CR multi-sensor networks
}

\author{
Zheng Dou, Chengzhuo Shi ${ }^{*}$ Y Yun Lin and Wenwen Li
}

\begin{abstract}
Motivated by the practical and accurate demand of intelligent cognitive radio (CR) sensor networks, a new modeling method of practical background noise and a novel sensing scheme are presented, where the noise model is the non-Gaussian colored noise based on $\alpha$ stable process and the sensing method is improved fractional low-order moment (FLOM) detection algorithm with balance parameter. First, we establish the non-Gaussian colored noise model through combining $\alpha$-distribution with a linear system represented by a matrix. And a fitting curve of practical noise data is given to verify the validity of the proposed model. Then we present a parameter estimation method with low complexity to obtain the balance parameter, which is an important part of the detection algorithm. The balance parameter-based FLOM (BP-FLOM) detector does not require any a priori knowledge about the primary user signal and channels. Monte Carlo simulations clearly demonstrate the performance of the proposed method versus the generalized signal-to-noise ratio, the characteristic exponent $\alpha$, and the number of detectors in sensing networks.
\end{abstract}

Keywords: Non-Gaussian colored noise model, BP-FLOM, Estimation, Practical noise data, CR multi-sensor networks

\section{Introduction}

The cognitive radio (CR) nodes with sensing and adaptive abilities have been recognized as a promising solution [1] to realize the next-generation intelligent sensing networks; the key ideas behind detector nodes lie in sensing spectrum information accurately under the practical noise background. Gaussian white noise are typically used to model practical noise processes that affect digital sensing systems [1], such as the multi-radar system and underwater acoustic detection system. In practice, however, Gaussian models reveal difficulties in fitting data that often have distinct spiky and impulsive characteristics leading them deviate from Gaussian distributions which is known as non-Gaussian [2]. Such non-Gaussian makes the common Gaussian assumption not valid for traditional spectrum sensing [3]. One of the most important challenges in sensor networks is to detect as quickly and reliably as possible the absence or presence of the signal in complex radio environments such as those characterized by non-Gaussian noises. Thus, the effective model of

\footnotetext{
*Correspondence: killformer1@gmail.com

School of Electronics and Information Engineering, Harbin Engineering

University, Nantong Street No.145, Nangang District, 150001 Harbin, China
}

practical noise and the realization of accurate detection are the main problems to be solved.

Non-Gaussian noise impairments may result from human factors and the natural factors, such as man-made impulse noise, electromagnetic equipment, atmospheric storms, and out of band spectral leakage $[4,5]$. The nonGaussian noise model should not only take into account its exact description of the nature for the noise, but also the simplicity of the calculation. Large measured data show that the probability density distribution of the impulse noise process is similar to the Gaussian process: symmetrical, smooth, and bell-shaped, but its tail is heavier than the Gaussian distribution [6]. The Gaussian mixture density (GMD) [7], centered generalized Gaussian density (GDD) [8], and the symmetric $\alpha$-stable $(\mathrm{S} \alpha \mathrm{S})$ density distribution [9] are most common models in recent years. The $S \alpha S$ distribution has proved to be the most promising model to fit many impulsive noise processes in communications channels, and, in fact, includes the Gaussian density as a special case [10]. Due to its good performance, the $\alpha$-stable distribution is used to fit the noise and interference in cognitive radio multi-sensor networks $[11,12]$, but the colored noise is not considered. 
Many spectrum sensing schemes for non-Gaussian noise has been presented in many literatures. The performances of Cauchy detector and global optimal detector are not ideal in the non-Gaussian noise [13, 14]. PolarityCoincidence-Array (PCA)-based spectrum sensing is proposed in [15], a significant performance enhancement is achieved by the PCA detector, but the prior knowledge such as the variance of the noise and the PU signal cyclic frequency are also needed in the algorithm, which is difficult in practical system. The Lp-Norm Spectrum Sensing method for cognitive radio networks is presented in [16]. It does not require any prior knowledge about the nonGaussian noise, but the condition that the noise do not have second-order statistics and high-order statistics such as $\alpha$-stable distribution are not taken into account, there are limitations when applied. The authors proposed a novel FLOM-based detector for the detection of a primary user in the $S \alpha S$ noises that can significantly enhance the detection performance compared to other detector algorithms $[4,17]$. But it is same to other detection algorithms, it is applicable only in the background non-Gaussian white noise. In addition, the algorithm relies on the characteristic exponent $\alpha$ of the $\mathrm{S} \alpha \mathrm{S}$ distribution, but it is difficult to obtain in the actual detection process.

Although non-Gaussian noise in sensing networks are given a variety of modeling and spectrum detection algorithm [18], most of them remain in the simulation and limit to white noise. We sample the practical noise data in laboratory environment with CR equipment USRP X-300 and analyze the power spectrum density. The result shows that it is not flat, which means the CR sensing system is working in the background of colored noise. That is why the performance of the algorithm mentioned above is declined when applied to practice, as pointed in $[19,20]$. Therefore, we propose a novel model to describe the non-Gaussian colored noise and present a new detection method to sensing signals. What is more, at special values, Gaussian white noise or non-Gaussian white noise can be included, which is more widely used in the practical system.

In this paper, we first fit the curve of practice noise data to study its characteristics and give a novel model to present the colored non-Gaussian noise through combining symmetric $\alpha$-distribution with a linear system represented by a matrix. Then we give an improved method to estimate the parameters (characteristic exponent $\alpha$ and dispersion $\gamma$ same to $\alpha$-stable density distribution) of the new distribution from a time series [21]. According to the estimation result, we propose a new sensing method of balance parameter-based fractional lower order moment (BP-FLOM), referred to as BP-FLOM detector. No prior knowledge is needed and the calculation is simplified. We also investigate the detection performance with different characteristic exponent $\alpha$ and the performance at different signal-to-noise ratios. In addition, multi-sensor performance is also simulated to verify the validity.

The remainder of this paper is organized as follows: In Section 2, we present the analysis of practical noise and establish the model of non-Gaussian noise. The estimation method for the new model is proposed in Section 3, and we give a new BP-FLOM algorithm based on the estimation result. Simulations results and analysis are presented in Section 4 and we conclude the paper in Section 5.

\section{Observation models and problem description}

In this section, a brief description of the most commonly models used in survival literature for the $C R$ sensing node is provided. They include the system model of CR networks [22] and the symmetric $\alpha$-stable density distribution [17]. And the new model is presented based on the analysis of practical noise data.

\subsection{System model}

Assume that the CR comprised of one Primer User (PU) and $M$ Secondary Users (SU). The received observation vector at the multi-sensors CR form the PU at time $n$ under each hypothesis (PU absent/present) is given by

$$
\begin{array}{rlrl}
\mathcal{H}_{0}: Y_{m}[n] & =\xi_{m}[n], & n & =0,1, \ldots, N-1 . \\
\mathcal{H}_{1}: Y_{m}[n] & =S_{m}[n]+\xi_{m}[n], n & =0,1, \ldots, N-1 .
\end{array}
$$

where $Y_{m}[n]=\left[y_{1}(n), y_{2}(n), \ldots, y_{m}(n)\right]^{T}$ is the sample of received signal by $\mathrm{m} t h$ detector at the time $n$, $N$ is the length of the sample sequence. $\xi_{m}[n]=$ $\left[\xi_{1}(n), \xi_{2}(n), \ldots, \xi_{m}(n)\right]^{T}$ is an additive background noise and $S_{m}[n]$ is primary signal to be detected. The primary signal is assumed to be random sequence of Gaussian distributions, and symmetric $\alpha$-stable distribution has proved to be a good way to describe the non-Gaussian noise [4].

The probability density function (PDF) of an $\alpha$ stable random variable cannot be given in closed form, but the characteristic function can always be given as followed [23]

$$
\phi(t)=\exp \left\{-\gamma|t|^{\alpha}+i \delta t\right\}
$$

where $\alpha \in(0,2]$ is the characteristic exponent, and it describes the tail of the distribution. The values $\alpha=2$ and $\alpha=1$ correspond to the Gaussian distribution and Cauchy distribution. The other two parameters are $\gamma>$ 0 for dispersion scale and $\delta \in R$ for location. Let $\mathrm{X} \sim$ $(\alpha, \beta, \gamma, \delta)$, the symmetric $\alpha$ stable $(\mathrm{S} \alpha \mathrm{S})$ distribution is given by $\mathrm{X} \sim(\alpha, 0,1,0)$ and only the $\mathrm{S} \alpha \mathrm{S}$ is considered in this paper. 


\subsection{Noise model}

The power spectral density of the practical noise is shown in Fig. 1. It is obvious that the power spectral density of practical noise is not flat. So the $\mathrm{S} \alpha \mathrm{S}$ distribution can not describe the practical noise perfectly as it is colored. To describe the practical noise accurately, we analyze the characteristics of $\mathrm{S} \alpha \mathrm{S}$ distribution as follows.

if $X(n), \forall n \in\{1,2, \ldots, N\}$ are independent and identically distributed (I.I.D) copies of $X$ and

$$
\sum_{n=1}^{N} a_{n} X^{(n)} \triangleq X_{n e w}
$$

where $N \in Z^{+}$and $a_{n}, c \in R$, then $X_{\text {new }}$ is the $S \alpha S$ distribution with same parameter $\alpha[17]$. According to the nature of colored noise that the noise in the sequence is correlated at each moment, we consider the colored noise to be a linear output driven by a white noise sequence. We propose a novel model for non-Gaussian colored noise as

$$
\begin{aligned}
\xi(n) & =X^{T}(n) \cdot A \\
& =\sum_{i=1}^{n} X_{i} \sim\left(\alpha, 0, a_{i} \gamma, 0\right) \\
& =X_{\text {nov }} \sim\left(\alpha, 0, \gamma_{\text {nov }}, 0\right)
\end{aligned}
$$

where $X[n]=[x(n), x(n-1)], \ldots, x(0)]^{T}$, is the sequence of $\mathrm{S} \alpha \mathrm{S}$ distribution. $A=\left[a_{1}, a_{2}, \ldots, a_{N}\right]$ is the linear transformation matrix. $\xi(n)=X_{n o v} \sim\left(\alpha, 0, \gamma_{n o v}, 0\right)$ is the colored noise sequence and has the same characteristic exponent to $X(n)$ according to [17]. In particular, when special parameter is taken as $A=\left[a_{1}, 0, \ldots, 0\right]$, the proposed model is the $\mathrm{S} \alpha \mathrm{S}$ distribution. The PDF of the new model based on $\mathrm{S} \alpha \mathrm{S}$ and different characteristic exponent $\alpha$ are plotted in Fig. 2. As expected, the heavy tail of non-Gaussian colored noise is consistent with the characteristics of the $\alpha$ distribution.

The power spectral density of the novel model is shown in Fig. 3, it can be concluded that it is very close to the practical noise that proves the validity of the model. As the $\mathrm{S} \alpha \mathrm{S}$ distribution has only the fractional lower moments, and its variance does not exist, the conventional signal-tonoise ratio is meaningless. So the signal-to-noise ratio for non-Gaussian colored noise is defined by mixed signalto-noise, we call it general signal-to-noise ratio (GSNR)

$$
G_{S N R} d B=10 \log _{10}\left(\frac{\sigma_{s}^{2}}{\gamma_{n o v}}\right)
$$

where $\sigma_{s}^{2}$ is the variance of Gaussian signal, and $\gamma_{n o v}$ is the dispersion scale of Gaussian colored noise. The GSNR will be applied to the subsequent simulation analysis.

\section{BP-FLOM-based spectrum sensing}

In this section, we propose a new spectrum sensing scheme, namely balance parameter-based fractional loworder moment (BP-FLOM) detector, for the non-Gaussian colored noise background. Adopted parameter estimation method associated with the proposed BP-FLOM method along with the potential of employing BP-FLOM detector in cooperative sensing is presented.

\subsection{Estimation of characteristic exponent $\alpha$ and $\gamma_{\text {nov }}$}

As introduced in Section 2, we assume that $\xi=a \xi_{1}+b \xi_{2}$, $\xi_{1}$ and $\xi_{2}$ are I.I.D sequence, the non-Gaussian colored noise $\xi$ has the same characteristic exponent $\alpha$ with $\mathrm{S} \alpha \mathrm{S}$, and a different dispersion scale $\gamma_{\text {nov }}$. To simplify the calculation, we do not consider the specific value of $\gamma_{n o v}$, and

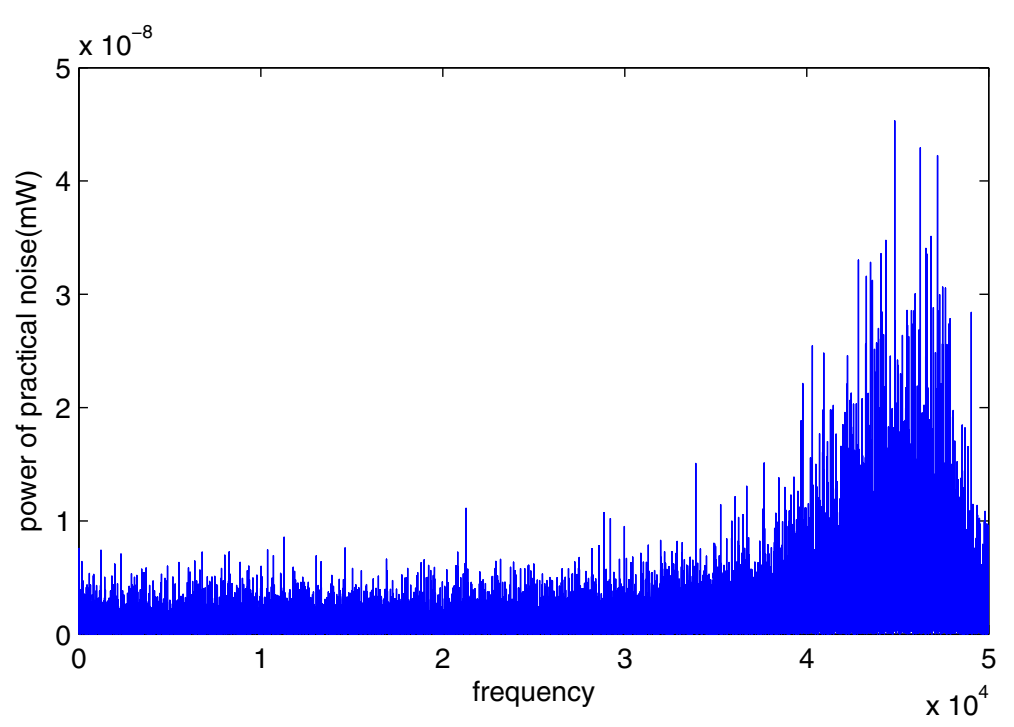

Fig. 1 Power spectral density of practical noise. The practical noise data are sampled in laboratory environment with the CR equipment USRP X-300 


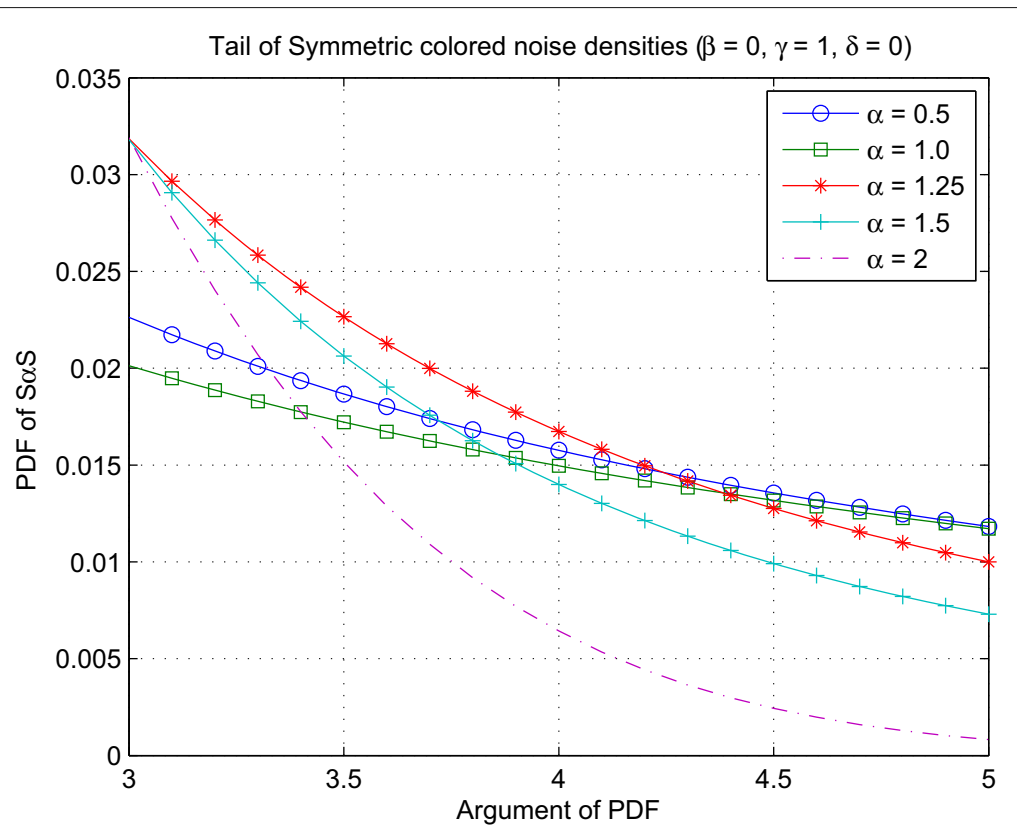

Fig. 2 PDF tails of non-Gaussian colored noise with different characteristic exponent $\alpha$

assume it is a known parameter. Then we improve the estimation method in [21], so it has a finite factional lower order moment for $a \xi_{1}+b \xi_{2}$

$$
E\left(\left|a \xi_{1}+b \xi_{2}\right|^{p}\right)=C_{1}(p, \alpha) \gamma_{\text {nov }}^{p / \alpha}, \quad \text { for } \quad 0<p<\alpha
$$

where $C_{1}(p, \alpha)=\frac{2^{p} \Gamma\left(\frac{p+1}{2}\right) \Gamma(1-p / \alpha)}{\sqrt{\pi} \Gamma(1-p / 2)}, 0<\alpha \leq 2, \gamma$ is the dispersion scale and $\Gamma(\cdot)$ is the gamma function. Then we obtained from (6)

$$
\log \gamma_{\text {nov }}=\frac{\alpha}{p} \log \frac{E\left(\left|a \xi_{1}+b \xi_{2}\right|^{p}\right)}{C_{1}(p, \alpha)}
$$

If $p$ th order of $\xi$ satisfies (6), we can write $\left.\mathrm{E}\left(\left|a \xi_{1}+b \xi_{2}\right|^{p}\right)\right)$ as $E\left(e^{p \log \left|a \xi_{1}+b \xi_{2}\right|}\right)$ and define a new variable $Z=\log \mid a \xi_{1}+$ $b \xi_{2} \mid$. Then

$$
E\left(|\xi|^{p}\right)=E\left(e^{p \log \left|a \xi_{1}+b \xi_{2}\right|}\right)=E\left(e^{p Z}\right), \quad 0<p<\alpha
$$

where $\mathrm{E}\left(e^{p Z}\right)$ is the moment-generating function of $Z$. The power series can be expressed by

$$
E\left(e^{p Z}\right)=\sum_{k=0}^{\infty} E\left(Z^{k}\right) \frac{p^{k}}{k !}
$$

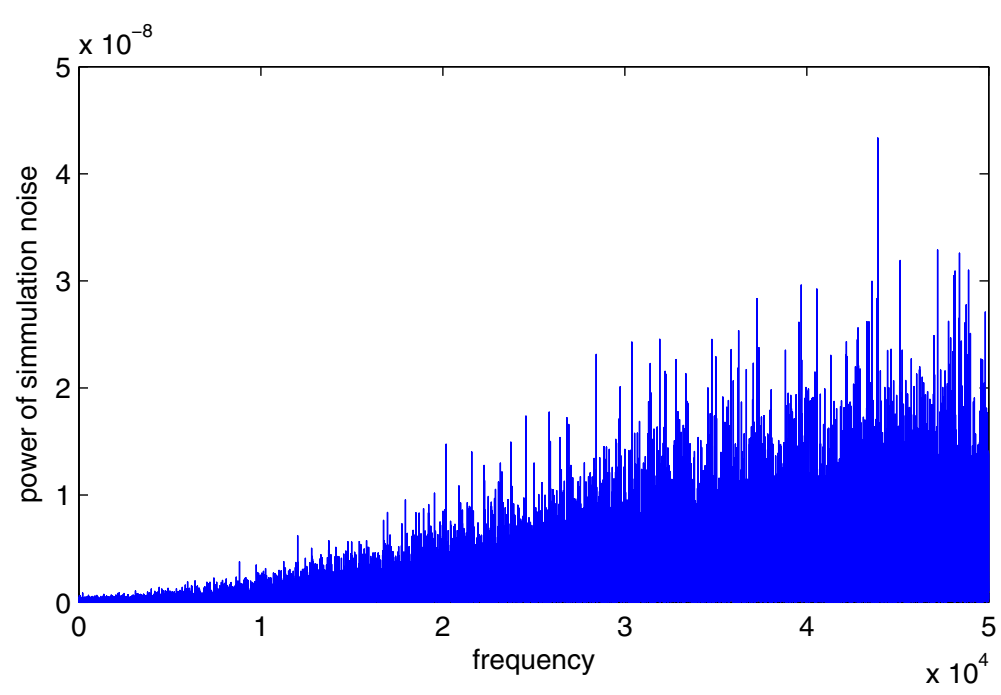

Fig. 3 Power spectral density of simulation colored noise. The data length is $10 \mathrm{e} 4$ and the Fs $=10 \mathrm{e} 4 \mathrm{MHz}, \alpha=1.8$ 
It is obvious that the moment of $Z$ is finite in any order, together with (6) we have

$$
E\left(Z^{k}\right)=\frac{d^{k}}{d p^{k}}\left(C_{1}(p, \alpha)\right) \gamma_{\text {nov }}^{p / \alpha}, \quad p=0
$$

Simplify the above equation according to [21]

$$
E\left(\log \left|a \xi_{1}+b \xi_{2}\right|\right)=C_{e}\left(\frac{1}{\alpha}-1\right)+\frac{1}{\alpha} \log \gamma_{n o v}
$$

where $C_{e}=0.57721566 \ldots$ is the Euler constant, $\alpha$ is the characteristic exponent, then combined (6) and (11) we have

$$
\begin{aligned}
E\left\{\left(\log \mid a \xi_{1}\right.\right. & \left.\left.+b \xi_{2} \mid-E\left[\log \left|a \xi_{1}+b \xi_{2}\right|\right]\right)^{2}\right\} \\
& =\frac{\pi^{2}}{6}\left(\frac{1}{\alpha^{2}}+\frac{1}{2}\right)
\end{aligned}
$$

Equation (12) can be used to estimate $\alpha$ in this way without calculating the value of $\gamma_{n o v}$, and it is easily obtained with (11).

Simulation result is given in Table 1 . As can be seen from the table, we can estimate the value of $\alpha$ exactly with a small relative error when the sampling of noise sequence is enough. The verification of the estimation method will be discussed in Section 4 .

\subsection{Spectrum sensing based on balance parameter}

We have known that the fractional low-order moment detection (FLOM) has a good performance under the $S \alpha \mathrm{S}$ distribution noise that is presented in [4], and it is more suitable than Cauchy detector. But when applied to the non-Gaussian colored noise background, the performance is declined. Based on the analysis in Section 2, we give the hypothesis A: The practical non-Gaussian colored noise is obtained by the linear transformation of the non-Gaussian white noise sequence obeying the $\mathrm{S} \alpha \mathrm{S}$ distribution.

Since the impulse response of linear transformation is difficult to compute without prior knowledge, we propose a simpler and more accurate approximation algorithm.

\subsubsection{Derivation of sensing threshold}

It is easy for us to obtain a $\mathrm{S} \alpha \mathrm{S}$ distribution sequence based on the parameter $\alpha_{e}, X_{k} \sim\left(\alpha_{e}, 0,1,0\right), \alpha_{e}$ is estimated by the estimation algorithm presented in Section 2, which is same to the practical noise sequence. Then, the sensing threshold of detector for the constructed sequence $X_{k} \sim\left(\alpha_{e}, 0,1,0\right)$, which is non-Gaussian white noise will be derived according to [4]. The detection statistic obtained in multi-user detection is :

$$
T_{F}=\frac{1}{M N} \sum_{m=1}^{M} \sum_{n=1}^{N}\left|X_{m}(n)\right|^{p_{e}}
$$

where $M$ is the number of detectors, and $N$ is the observation signal of each detector. $0<p_{e}<\alpha_{e} / 2$ is the order of the fractional moment, and it is the only parameter to be determined. Then make a comparison by statistics $T_{F}$ and threshold $\eta$, if $T_{F}>\eta$, it is considered that the primary signal is present, otherwise it is absent. The detection signal does not require any priori information such as the channel gain and primary signal. It is practical and provides a scheme for the detection of non-Gaussian colored noise. The expressions for the probability of false alarm and the detection under the hypothesis $\mathcal{H}_{0}$ and $\mathcal{H}_{1}$ are derived, including the multi-detectors.

Under hypothesis $\mathcal{H}_{0}$, the mean of the $T_{F}$ is calculated by

$$
\mu_{0}=E\left[T_{F} \mid \mathcal{H}_{0}\right]=\frac{1}{M N} E\left[\sum_{m=1}^{M} \sum_{n=1}^{N}\left|X_{m}(n)\right|^{p_{e}}\right]
$$

According to the properties of $\alpha$ stable distribution, the fractional lower order moments of any $\mathrm{S} \alpha \mathrm{S}$ random variable $S$ can be represented by its characteristic index $\alpha$ and the dispersion scale $\gamma$ [24]

$$
E\left(|S|^{p}\right)=C(p, \alpha) \gamma^{p / \alpha}, \text { for } p<\alpha
$$

where

$$
C(p, \alpha)=\frac{2^{p+1} \Gamma\left(\frac{p+1}{2}\right) \Gamma(-p / \alpha)}{\alpha \sqrt{\pi} \Gamma(-p / 2)}
$$

Here, $\Gamma(\sigma)=\int_{0}^{\infty} x^{\sigma-1} e^{-x} d x$. Applying (15) and (16) to Eq. (14), it can be rewritten as

$$
\mu_{0}=\frac{1}{M} \sum_{m=1}^{M} C\left(p_{e}, \alpha_{e}\right) \gamma^{p_{e} / \alpha_{e}}
$$

Table 1 Estimation of $\alpha$. The estimation result of $\alpha$ with different sample size, where real value is 1.5 and 1.2

\begin{tabular}{lllll}
\hline Sample size & 1000 & 5000 & 10,000 & 50,000 \\
\hline Test result & 1.5301 & 1.4994 & 1.5088 & 1.5009 \\
Real value & 1.5 & 1.5 & 1.5 & 1.5 \\
Relative error & $2 \%$ & $0.4 \%$ & $0.59 \%$ & $0.6 \%$ \\
Test result & 1.2117 & 1.2030 & 1.1946 & 1.2021 \\
Real value & 1.2 & 1.2 & 1.2 & 1.2 \\
Relative error & $0.97 \%$ & $0.25 \%$ & $0.53 \%$ & $0.18 \%$ \\
\hline
\end{tabular}


And the variance of statistic under $\mathcal{H}_{0}$

$$
\begin{aligned}
\sigma_{0}^{2}= & E\left[\left(T_{F} \mid \mathcal{H}_{0}\right)^{2}-E^{2}\left[\left(T_{F} \mid \mathcal{H}_{0}\right)\right]\right] \\
= & \frac{1}{M^{2} N}\left\{\sum_{m=1}^{M} E\left[\left|V_{m}(n)\right|^{2 p_{e}}\right]\right. \\
& \left.-\sum_{m=1}^{M} E^{2}\left[\left|V_{m}(n)\right|^{p_{e}}\right]\right\}
\end{aligned}
$$

According to the central limit theorem, $T_{F}$ is a Gaussian random variable when $N$ is large enough. With the result $\mu_{0}$ in (17)and $\sigma_{0}^{2}$ in (18), the probability of false alarm is obtained

$$
P_{f a 1}=\left\{T_{F}>\eta_{t} \mid \mathcal{H}_{0}\right\}=Q\left(\frac{\eta_{t}-\mu_{0}}{\sqrt{\sigma_{0}^{2}}}\right)
$$

Thus, the sensing threshold for constructed sequence $X_{k} \sim\left(\alpha_{e}, 0,1,0\right)$ is then

$$
\eta_{t}=\sqrt{\sigma_{0}^{2}} Q^{-1}\left(P_{f a 1}\right)+\mu_{0}, \quad P_{f a 1} \leq \bar{P}_{f a 1}
$$

\subsubsection{Balance parameter-based FLOM detector}

The simulation curve of $\eta_{t}$ and the $P_{f a 1}$ for the $X_{k} \sim$ $\left(\alpha_{e}, 0,1,0\right)$ constructed in the previous section can be fitted easily. When the $P_{f a 1}$ is determined, $\eta_{t}$ can be obtained by (21). But if we use this value as standard sensing threshold, detection is invalid. So, another statistics $T_{p}$ is needed to calculate the balance parameter.

According to hypothesis A, we assume that $\xi(n)$ is the non-Gaussian noise sequence under $\mathcal{H}_{0}$, a different statistics of $\xi(n)$ can be obtained.

$$
T_{P}=\frac{1}{M N} \sum_{m=1}^{M} \sum_{n=1}^{N}\left|\xi_{m}(n)\right|^{p_{\bar{m}}}
$$

When the sampling sequence $N$ is large, according to the central limit theorem, the statistical values of the two sequences tend to be stable value. Then we have the equation

$$
\eta_{p}=\eta_{t} \cdot \Delta
$$

where $\Delta=T_{F} / T_{P}$, we call it the balance parameter. $\eta_{p}$ is the sensing threshold for practical sensing node. In particular, when vector $\mathrm{A}$ in Section 2.2 is $[1,0, \ldots, 0], \Delta=1$ and $\eta_{t}=\eta_{p}$. To obtain the false alarm probability and detection probability, the $\sigma_{p i}^{2}$ and $\mu_{p i}$ is needed, $i=0,1$.
Substituting (13) to (19), and assume $X_{m}(n)=a \xi_{1}(n)+$ $b \xi_{2}(n)$, combined (15), we obtain

$$
\begin{aligned}
\sigma_{p 0}^{2}= & E\left[\left(\frac{1}{M N} \sum_{m=1}^{M} \sum_{n=1}^{N}\left[\left|X_{m}(n)\right|^{p_{e}}\right]\right)^{2}\right] \\
& -\left\{\frac{1}{M N} \sum_{m=1}^{M} \sum_{n=1}^{N} E\left[\left|X_{m}(n)\right|^{p_{e}}\right]\right\}^{2} \\
= & \frac{1}{M^{2} N^{2}}\left\{N E\left[\sum_{m=1}^{M}\left|X_{m}(n)\right|^{2 p_{e}}\right]\right. \\
& +\sum_{\text {Case: } a}^{M} \sum_{n, j=1}^{N} E\left[\left|X_{m}(n)\right|^{p_{e}}\left|X_{i}(j)\right|^{p_{j}}\right] \\
& -N \sum_{m=1}^{M} E^{2}\left[\left|x_{m}(n)\right|_{m}^{p_{m}}\right] \\
& -\sum_{\text {Case: } b n, j=1}^{M} E\left[\left|X_{m}(n)\right|^{p_{e}}\left|X_{i}(j)\right|^{p_{j}}\right] \\
= & \frac{1}{M^{2} N}\left\{\sum_{m=1}^{M} E\left[\left|\xi_{1}(n)+b \xi_{2}(n)\right|^{2 p_{e}}\right]\right. \\
& \left.-\sum_{m=1}^{M} E^{2}\left[\left|\xi_{1}(n)+b \xi_{2}(n)\right|^{p_{e}}\right]\right\}
\end{aligned}
$$

Noting that both case $a$ and case $b$ are $m, i=1, m \neq i$ or $n \neq j$. As $p_{e}<\alpha_{e} / 2$, using (15) to (24) is then

$$
\begin{aligned}
\sigma_{P 0}^{2}= & \frac{1}{M^{2} N} \sum_{m=1}^{M}\left[C\left(2 p_{e}, \alpha_{e}\right) \gamma_{e}^{2 p_{e} / \alpha_{e}}\right. \\
& \left.-\left(C\left(p_{e}, \alpha_{e}\right) \gamma_{e}^{p_{e} / \alpha_{e}}\right)^{2}\right]
\end{aligned}
$$

where $\gamma_{e}=\gamma_{\text {nov }}$ and

$$
\mu_{p 0}=\frac{1}{M} \sum_{m=1}^{M} C\left(p_{e}, \alpha_{e}\right) \gamma_{e}^{p_{e} / \alpha_{e}}
$$

Similarly, the mean $\mu_{1}$ and variance $\sigma_{1}^{2}$ of $T_{F}$ under $\mathcal{H}_{1}$ are derived then

$$
\mu_{p 1}=E\left[T_{F} \mid \mathcal{H}_{1}\right]=\mu_{0}+\sum_{m=1}^{M} \phi_{m, 0}
$$

where

$$
\phi_{m, 0}=\frac{\sigma_{s}^{2} p_{e}\left(p_{e}-1\right) C\left(p_{e}-2\right), \alpha_{e}}{2 M} \gamma_{e}^{\left(p_{e}-2\right) / \alpha_{e}}
$$

And the variance under $\mathcal{H}_{1}$

$$
\begin{aligned}
\sigma_{p 1}^{2} & =E\left[\left(T_{F} \mid \mathcal{H}_{1}\right)^{2}-E^{2}\left[\left(T_{F} \mid \mathcal{H}_{1}\right)\right]\right] \\
& =\sigma_{p 0}^{2}+\frac{1}{N}\left(\frac{\sigma_{s}}{M}\right)^{2} \sum_{m=1}^{M} \phi_{m, 1}
\end{aligned}
$$


where

$$
\begin{aligned}
& \phi_{m, 1}=p_{e}\left(2 p_{e}-1\right) C\left(2 p_{e}-2, \alpha_{e}\right) \gamma_{e}^{2\left(p_{e}-1\right) / \alpha_{e}} \\
& -p_{e}\left(p_{e}-1\right) C\left(p_{e}, \alpha_{e} \gamma_{e}^{p_{e} / \alpha_{e}}\right)\left(2 p_{e}-2, \alpha_{e}\right) \gamma_{e}^{\left(p_{e}-2\right) / \alpha_{e}}
\end{aligned}
$$

According to the central limit theorem, $T_{F}$ is a Gaussian random variable when $N$ is large enough. With the result $\mu_{p 0}$ in (24)and $\sigma_{p 0}^{2}$ in (25), the probability of false alarm is obtained

$$
P_{f a}=\left\{T_{P}>\eta_{P} \mid \mathcal{H}_{0}\right\}=Q\left(\frac{\eta_{t} \Delta-\mu_{p 0}}{\sqrt{\sigma_{p 0}^{2}}}\right)
$$

and detection probability with $\mu_{p 1}$ and $\sigma_{p 1}^{2}$

$$
P_{d}=\left\{T_{P}>\eta_{P} \mid \mathcal{H}_{0}\right\}=Q\left(\frac{\eta_{t} \Delta-\mu_{p 1}}{\sqrt{\sigma_{p 1}^{2}}}\right)
$$

where $\mu_{p i}$ and $\sigma_{i}^{2}(i=1,2)$ are the mean and variance of practical noise sequence. The expression of probability detection can be deduced by the combination of (28) and (29)

$$
P_{d}=Q\left(\frac{\sqrt{\left(\sigma_{0}^{2}\right)} Q^{-1}\left(P_{f a}\right)+\mu_{p 0}-\mu_{p 1}}{\sqrt{\sigma_{p 1}^{2}}}\right)
$$

Substituting (26 - 28) into (31)

$$
P_{d}=Q\left(\frac{\sqrt{\left(\sigma_{0}^{2}\right)} Q^{-1}\left(P_{f a}\right)-\sum_{m=1}^{M} \phi_{m, 0}}{\sqrt{\sigma_{0}^{2}+\frac{\sigma_{s}^{2}}{M^{2} N} \sum_{m=1}^{M} \phi_{m, 1}}}\right)
$$

The optimal $P_{d}$ can be obtained by searching for the order vector $p_{\bar{m}}$ because $\sigma_{0}^{2}, \phi_{m, 0}$ and $\phi_{m, 1}$ are all related to $p_{\bar{m}}$. Then numerical computation of (31) will be conducted in Section 4 as well as Monte Carlo simulations to validate our algorithm.

\section{Simulation results}

In this section, the simulation result will be discussed to evaluate the performance of the novel model for nonGaussian colored noise as well as the BP-FLOM detector.

\subsection{Effectiveness of noise models}

First, we investigate the effectiveness of the non-Gaussian colored noise model as well as the parameter estimated method. The comparison for power spectral density is presented in Section 2, the proposed non-Gaussian colored noise model has the same statistical properties to the practical noise.

Then we construct a new non-Gaussian colored noise sequence $\xi(n)$, the characteristic exponent $\alpha=1.25$, the linear transformation matrix $\mathrm{A}=\left[\begin{array}{l}0.9-1.25 \\ 0.7\end{array}\right]$, sample

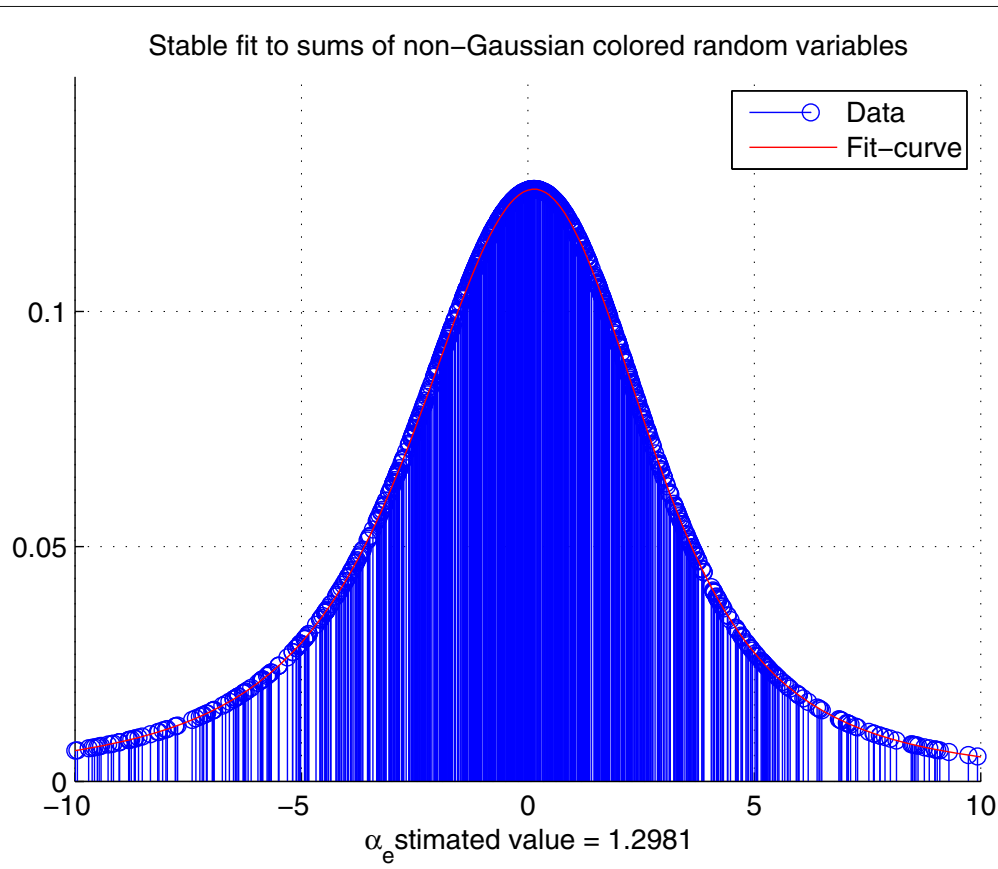

Fig. 4 The PDF and fit curve of non-Gaussian colored sequence. The blue part are the data distribution, the red line is the fit-curve with estimated parameters 
Table 2 Estimation of result. The estimation result of $\alpha$ with different sample size, where real value is 1.5

\begin{tabular}{lllll}
\hline Estimation times & 1 & 2 & 3 & 4 \\
\hline Test result & 1.2426 & 1.2513 & 1.2486 & 1.2509 \\
Real value & 1.25 & 1.25 & 1.25 & 1.25 \\
Relative error & $0.59 \%$ & $0.14 \%$ & $0.11 \%$ & $0.07 \%$ \\
\hline
\end{tabular}

size is set to $L=100,000$. As shown in Fig. 4, the distribution of the data follow the shape of the probability density distribution.

Next we estimate the parameters of the sequence to draw a new fit curve. It is obvious that the fitted curve is consistent with the data distribution. The estimation result is shown in Table 2 . The result of parameter estimation is very close to the parameter $\alpha$ of the constructed sequence, and the estimation relative error is very small.

In conclusion, the proposed model has the same statistical characteristics, the practical noise is estimated, and the reconstructed data are consistent with the actual ones. all the analysis above show that non-Gaussian colored noise model we proposed can describe the real noise accurately.

\subsection{The performance of BP-FLOM detector}

We assume that the primary signal is Gaussian with 0 mean, variance $\sigma_{s}^{2}$, the noise background is the nonGaussian colored noise based on I.I.D $\mathrm{S} \alpha \mathrm{S}$ with dispersion scale $\gamma=1$. We set the sample size $N=1000$, and the simulation results are achieved by 20,000 Monte Carlo simulations.
Figure 5 shows the curves of the detection threshold versus the probability of false alarm, and different values for the single-node detection parameters. Curves labeled with "theory" are calculated according to expression (28), curves labeled with "simulation" are obtained statistic and detection threshold. In the case of cooperative detection, we set $\alpha_{1}=2, p_{1}=0.7$ for the first detector and $\alpha_{2}=$ $0.8, p_{2}=0.33$. Figure 6 shows $M=2$ for cooperative detection, the comparison is also displayed. It proves that the simulation is consistent with the theory.

Figure 7 shows the comparison of the three sets of simulation results, and the range of GSNR is $-15 \sim$ $5 \mathrm{~dB}$. Three different characteristic exponents and corresponding fractional lower order are presented to make a comparison between FLOM detector and the BP-FLOM we proposed. When the characteristic component $\alpha=2$, it is the Gaussian colored noise and the performance of the two algorithm is close to each other. For $\alpha=1.5$ and $\alpha=0.8$, our proposed detector has a better detection performance than the FLOM detector under all levels of GSNR. For instance, when GSNR $=-4 \mathrm{~dB}, P_{f} a=0.1$ and $\alpha=1.5$, the probability of detection of our detector is $71.4 \%$, but that of the FLOM detector is $42 \%$ only,

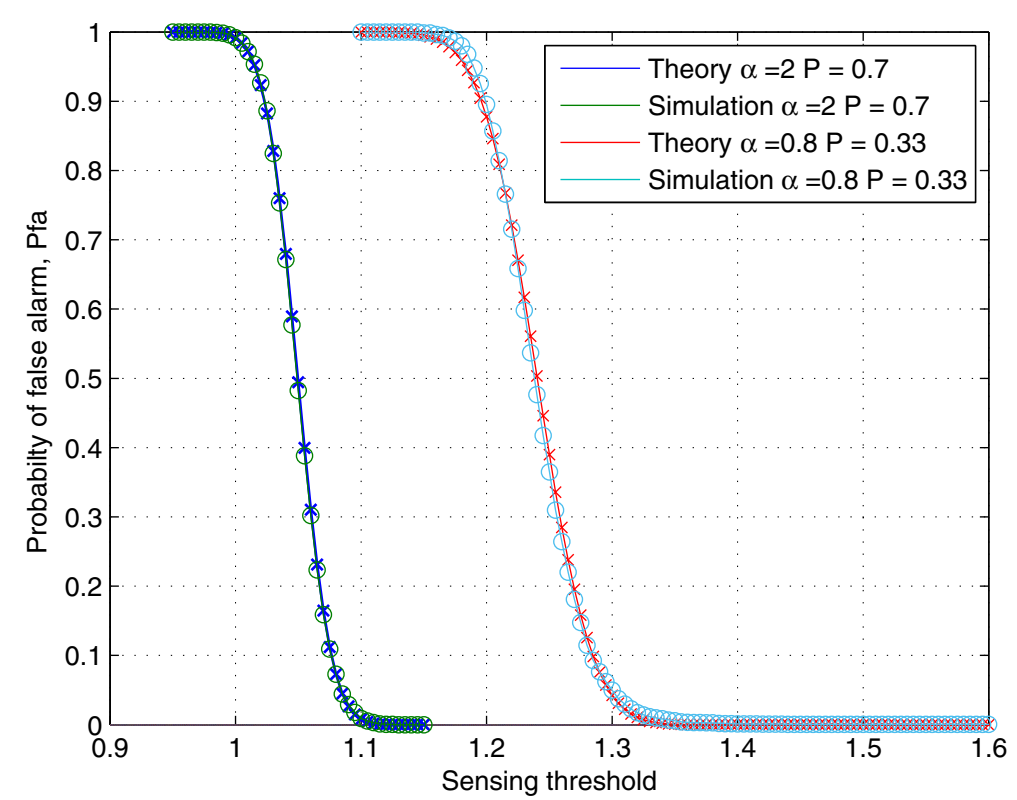

Fig. 5 Probability of false alarm versus detection threshold of single detector for different values of $\alpha$, $p$. The comparison of theory and simulation result are displayed 


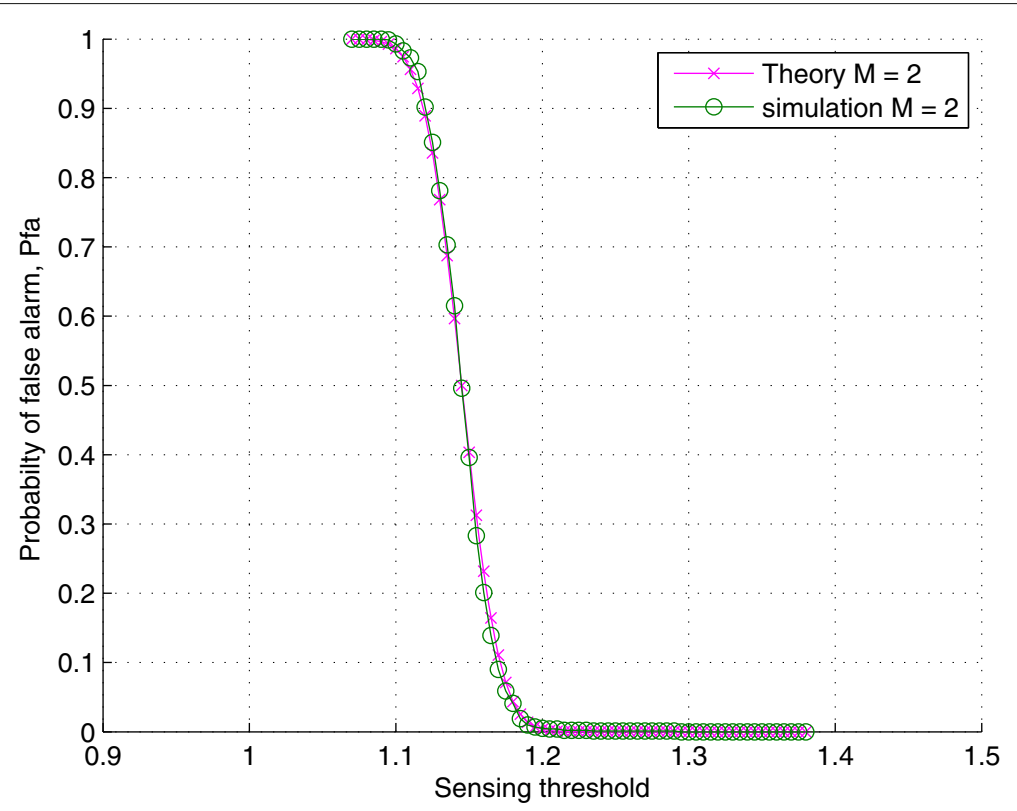

Fig. 6 Probability of false alarm versus detection threshold of a multi-detector. The number of detector is 2, and the comparison of theory and simulation result are displayed

which fails to detect the primary signal. But the detection is effective only under a large GSNR when $\alpha=0.8$. In summary, the performance of BP-FLOM detector is better than FLOM detector in the background of non-Gaussian colored noise.

Figure 8 shows the performance of the BP-FLOM detector under single detection and multi-detection; it is obvious that the performance of multi-detection is much better than that of single detection. For example, the probability of detection of multi-detection is $75 \%$ while the single detection is $42 \%$, under the same condition $P_{f} s=$ 0.1 .

Figure 9 shows the ROC curves of the BP-FLOM detector, both the simulation and theory curves. The simulation result are obtained with different values of $p=0.33$ and $p=0.7$ under two values of GSNR $(-5 \mathrm{~dB}$ and $-10 \mathrm{~dB})$.

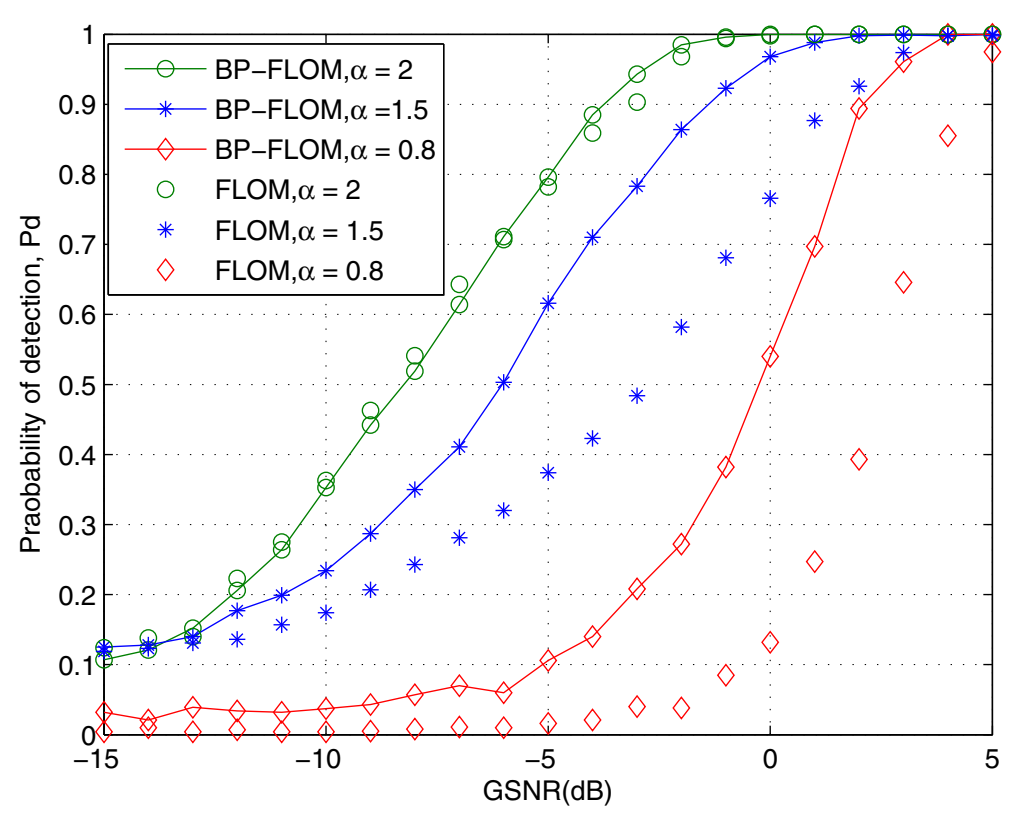

Fig. 7 Probability of false alarm versus detection threshold of BP-FLOM detector for different values of $\alpha$, $p$, and M 


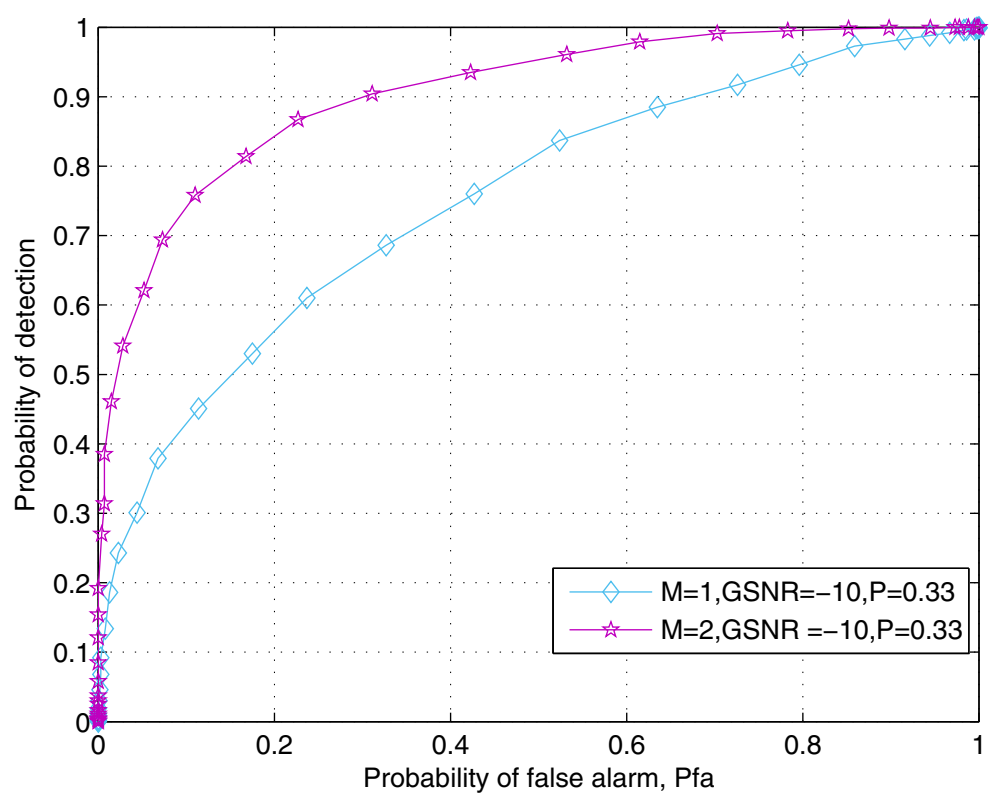

Fig. 8 ROC of BP-FLOM detector with different number of detectors. $M=2$ and $M=1$ represent different number of detectors for sensing

The theory curves are obtained with (31) under the same conditions. As shown in the figure, our proposed detector performs better for smaller values of $p$. For example, the probability of detection is $70 \%$ with $p=0.33$ while $37 \%$ with $p=0.7$, under the same GSNR $=-10 \mathrm{~dB}$.

\section{Conclusions}

This paper propose a novel non-Gaussian noise model based on the analysis of practical noise, and present a balance parameter-based fractional low-order moment (BP-FLOM) detector. It was shown that although the BP-FLOM scheme exhibits an approximately identical detection performance with the FLOM detector when it is Gaussian colored noise, performance is better when the background is the non-Gaussian colored noise, and the multi-detection performance is much better than FLOM detector and single detector. Simulation results, as well as the presented analysis, conform to the superior

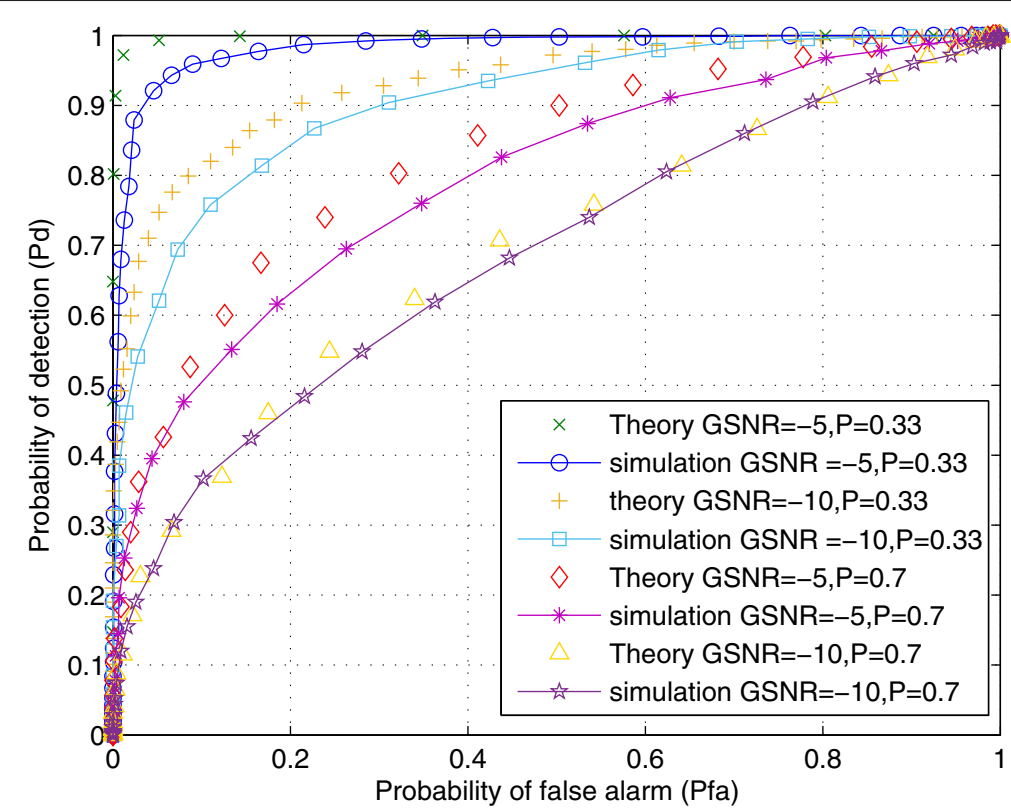

Fig. 9 ROC of BP-FLOM detector with multi-detectors. Performance in different conditions when GSNR $=-10 \mathrm{~dB}$ or $-5 \mathrm{~dB}, p=0.33$ or 0.7 , the theory and simulation are all displayed 
performance of the proposed balance parameter-based sensing scheme relative to competing solutions, in particular, the balance parameter is self adjusted when the noise sequence changed, which is practical for the system.

\section{Acknowledgements}

This work was supported by the National Nature Science Foundation of China (no. 61301095), National Nature Science Foundation of China (no. 61671167).

\section{Authors' contributions}

CS proposed the non-Gaussian colored noise based on S $\alpha$ S distribution and presented a new detection method of balance parameter-based FLOM detector. CS completed all the computer simulation and wrote the whole paper as well as the proofreading. All authors read and approved the final manuscript.

\section{Competing interests}

The authors declare that they have no competing interests.

\section{Publisher's Note}

Springer Nature remains neutral with regard to jurisdictional claims in published maps and institutional affiliations.

Received: 18 July 2017 Accepted: 7 November 2017

Published online: 16 November 2017

\section{References}

1. M Bkassiny, AL De Sousa, SK Jayaweera, Wideband spectrum sensing for cognitive radios in weakly correlated non-gaussian noise. IEEE Commun. Lett. 13, 266-267 (1996)

2. JMS Proakis, Digital Communications. (McGraw-Hill higher education, New York, 2007)

3. M Bkassiny, SK Jayaweera, Robust, non-gaussian wideband spectrum sensing in cognitive radios. IEEE Trans. Wirel. Commun. 13, 6410-6421 (2014)

4. BC Xiaomei Zhu, W-P Zhu, Spectrum sensing based on fractional lower order moments for cognitive radios in -stable distributed noise. Signal Process. 11, 94-105 (2015)

5. TM Taher, MJ Misurac, JL LoCicero, DR Ucci, Microwave oven signal interference mitigation for wi-fi communication systems. IEEE Consum. Commun. Netw. Conf. 13, 67-68 (2008)

6. G Laguna-Sanchez, M Lopez-Guerrero, On the use of alpha-stable distributions in noise modeling for plc. IEEE Trans. Power Deliv. 30, 1863-1870 (2015)

7. Y Zhao, X Zhuang, S-J Ting, Gaussian mixture density modeling of non-gaussian source for autoregressive process. IEEE Trans. Sig. Process. 4, 894-903 (1995)

8. MS Allili, N Baaziz, M Mejri, Texture modeling using contourlets and finite mixtures of generalized gaussian distributions and applications. IEEE Trans. Multimed. 16, 772-784 (2014)

9. F Wen, Diffusion least-mean p-power algorithms for distributed estimation in alpha-stable noise environments. Electron. Lett. 49, 1355-1356 (2013)

10. K Pelekanakis, M Chitre, Adaptive sparse channel estimation under symmetric alpha-stable noise. IEEE Trans. Wirel. Commun. 13, 3183-3195 (2014)

11. X Yang, AP Petropulu, Co-channel interference modeling and analysis in a poisson field of interferers in wireless communications. IEEE Trans. Signal Process. 1, 64-76 (2003)

12. MZ Win, PC Pinto, LA Shepp, A mathematical theory of network interference and its applications. Proc. IEEE. 97, 205-230 (2009)

13. EE Kuruoglu, WJ Fitzgerald, PJW Rayner, Near optimal detection of signals in impulsive noise modeled with a symmetric alpha-stable distribution. IEEE Commun. Lett. 2, 282-284 (1998)

14. HG Kang, I Song, S Yoon, YH Kim, A class of spectrum-sensing schemes for cognitive radio under impulsive noise circumstances: Structure and performance in nonfading and fading environments. IEEE Trans. Veh. Technol. 59, 4322-4339 (2010)
15. TWimalajeewa, PK Varshney, Polarity-coincidence-array based spectrum sensing for multiple antenna cognitive radios in the presence of non-gaussian noise. IEEE Trans. Wirel. Commun. 10, 2362-2371 (2011)

16. F Moghimi, A Nasri, R Schober, Adaptive I-p norm spectrum sensing for cognitive radio networks. IEEE Trans. Commun. 59, 1934-1945 (2011)

17. A Mahmood, M Chitre, MA Armand, On single-carrier communication in additive white symmetric alpha-stable noise. IEEE Trans. Commun. 62 , 3584-3599 (2014)

18. D Middleton, Non-gaussian noise models in signal processing for telecommunications: new methods an results for class a and class b noise models. IEEE Trans. Inf. Theory. 45, 1129-1149 (1999)

19. G Laguna-Sanchez, M Lopez-Guerrero, On the use of alpha-stable distributions in noise modeling for plc. IEEE Trans. Power Deliv. 30, 1863-1870 (2015)

20. G Bansal, MJ Hossain, P Kaligineedi, H Mercier, C Nicola, U Phuyal, MM Rashid, KC Wavegedara, Z Hasan, M Khabbazian, VK Bhargava, in AFRICON 2007. Some research issues in cognitive radio networks (IEEE, 2007), pp. 1-7

21. $\mathrm{X} M \mathrm{Ma}, \mathrm{CL}$ Nikias, Parameter estimation and blind channel identification in impulsive signal environments. IEEE Trans. Signal Process. 43, 266-267 (1995)

22. A Margoosian, J Abouei, KN Plataniotis, An accurate kernelized energy detection in gaussian and non-gaussian/impulsive noises. IEEE Trans. Signal Process. 63, 5621-5636 (2015)

23. WJ Szajnowski, JB Wynne, Simulation of dependent samples of symmetric alpha-stable clutter. IEEE Signal Process. Lett. 8, 151-152 (2001)

24. GA Tsihrintzis, CL Nikias, Performance of optimum and suboptimum receivers in the presence of impulsive noise modeled as an alpha-stable process. IEEE Trans. Commun. 43, 904-914 (1995)

\section{Submit your manuscript to a SpringerOpen ${ }^{\ominus}$ journal and benefit from:}

- Convenient online submission

- Rigorous peer review

- Open access: articles freely available online

- High visibility within the field

- Retaining the copyright to your article

Submit your next manuscript at $\mathbf{s p r i n g e r o p e n . c o m ~}$ 